\title{
OS EFEITOS DOS MAPAS CONCEITUAIS NA APRENDIZAGEM DOS ESTUDANTES UNIVERSITÁRIOS
}

\author{
THE EFFECTS OF CONCEPTUAL MAPPING IN \\ UNIVERSITY STUDENTS' LEARNING \\ LOS EFECTOS DE LOS MAPAS CONCEPTUALES EN EL APRENDIZAJE \\ DE LOS ESTUDIANTES UNIVERSITÁRIOS \\ Cristiane Tolentino Machado ${ }^{1}$, Ana Amélia Carvalho

\begin{abstract}
RESUMO
Introdução: No ensino superior, os estudantes necessitam de desenvolver habilidades cognitivas essenciais para a sua formação acadêmica. Os mapas conceituais são considerados ferramentas educacionais para promover uma aprendizagem significativa desenvolvendo nos estudantes o pensamento crítico, a capacidade de resolução de problemas e a compreensão profunda dos conceitos. Métodos: Foram selecionadas e analisadas as publicações de 1992 a 2017 que utilizaram os mapas conceituais no ensino superior. Resultados: Este artigo descreve os efeitos dos mapas conceituais nas atividades acadêmicas dos estudantes universitários, bem como identifica as suas finalidades e relevância para a promoção da aprendizagem no ensino superior. Nos estudos analisados, são identificados e discutidos os desafios para a integração dos mapas conceituais nas práticas acadêmicas. Conclusão: Apesar de algumas limitações, os mapas conceituais são em geral bem aceitos pelos estudantes como facilitares do processo de aprendizagem. Os mapas conceituais são ferramentas importantes para auxiliar na aprendizagem de estudantes universitários em várias áreas de conhecimento
\end{abstract}

PALAVRAS-CHAVE: Aprendizagem significativa. Ensino superior. Estudantes universitários. Mapas conceituais.

\begin{abstract}
Introduction: In higher education, students need to develop cognitive skills essential to their academic training. Conceptual maps are considered educational tools to promote meaningful learning by developing in students critical thinking, problem-solving ability, and deep understanding of concepts. Methods: Publications from 1992 to 2017 that used the concept maps in higher education were selected and analyzed. Results: This paper describes the effects of conceptual mapping used by students in their academic activities, as well as identifies their purposes and relevance to the promotion of learning in higher education. The conceptual maps also present challenges for their integration into the academic practices that are identified and discussed. Conclusion: Despite the limitations, conceptual maps are generally well accepted by students as facilitators during the learning process. Conceptual maps are important tools to assist in the learning of students in various areas of knowledge.
\end{abstract}

KEYWORDS: Concept maps. Higher education. Meaningful learning. University students.

\section{RESUMEN}

Introducción: En la enseñanza superior, los estudiantes necesitan desarrollar habilidades cognitivas esenciales para su formación académica. Los mapas conceptuales se consideran herramientas educativas para promover un aprendizaje significativo desarrollando en los estudiantes el pensamiento crítico, la capacidad de resolución de problemas y la comprensión profunda de los conceptos. Métodos: Las publicaciones de 1992 a 2017 que utilizaron los mapas conceptuales en la enseñanza superior fueron seleccionadas y analizadas. Resultados obtenidos: Este artículo describe los efectos de los mapas conceptuales utilizados por los estudiantes

\footnotetext{
${ }^{1}$ Doutora em Biologia Celular - Universidade Federal de Minas Gerais (UFMG) - Belo Horizonte, MG - Brasil. Professora - Universidade Federal dos Vales do Jequitinhonha e Mucuri (UFVJM) - Diamantina, MG - Brasil. Email: cristiane.tolentino@gmail.com

${ }^{2}$ Doutora em Educação - Universidade do Minho (UM) - Braga, Portugal. Professora Catedrática na Universidade de Coimbra (UC) - Faculdade de Psicologia e de Ciências da Educação - Coimbra, Portugal. Email: anaameliac@fpce.uc.pt
}

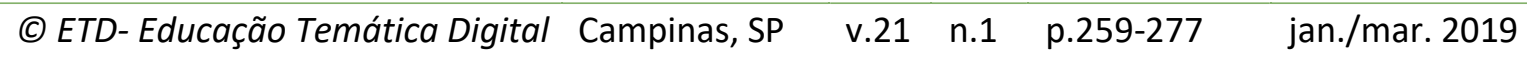


universitarios en sus actividades académicas, así como identifica sus propósitos y relevancia para la promoción del aprendizaje en la enseñanza superior. Los mapas conceptuales también presentan desafíos para su integración en las prácticas académicas que se identifican y se discuten. Conclusión: A pesar de las limitaciones, los mapas conceptuales son generalmente bien aceptados por los estudiantes como facilitadores del proceso de aprendizaje. Los mapas conceptuales demostraron ser herramientas importantes para auxiliar en el aprendizaje de estudiantes universitarios en varias áreas de conocimiento.

PALABRAS CLAVE: Aprendizaje significativo. Enseñanza superior. Estudiantes universitários. Mapas conceptuales.

\section{INTRODUÇÃO}

Baseados na epistemologia construtivista e na teoria de aprendizagem significativa de Ausubel (1960), os mapas conceituais foram desenvolvidos por Joseph D. Novak como uma ferramenta para organizar e representar o conhecimento. Trata-se de representações esquemáticas de conceitos ligados entre si por unidades semânticas denominadas proposições. Os conceitos aparecem nos nós do gráfico interligados pelas unidades semânticas que conectam dois ou mais conceitos. A organização dos conceitos deve ser feita de forma que haja uma hierarquia, ou seja, os conceitos mais gerais e inclusivos devem aparecer na parte superior do mapa e os conceitos mais específicos e menos inclusivos, na parte inferior (NOVAK; GOWIN, 1984). Ao tornarem mais claras as conexões entre os conceitos, os mapas conceituais colocam-se como um instrumento adequado para estruturar o conhecimento que está sendo construído, facilitando aos estudantes compreenderem o conteúdo e relacionarem novos conhecimentos com os que já possuem. Sua elaboração promove a aprendizagem significativa e evita a aprendizagem exclusivamente pela memorização (GONZÁLEZ et al., 2008). Assim, auxiliam os estudantes a estabelecerem conexões entre os conhecimentos teóricos envolvidos na apreciação de um problema, o que pode ter importante reflexo na prática profissional (AKINSANYA; WILLIAMS, 2004). Os mapas conceituais são uma ferramenta valiosa de aprendizagem e têm demonstrado efeitos positivos no ensino superior para aprender, ensinar e avaliar (RITCHHART; TURNER; HADAR, 2009). Constituem uma importante estratégia para incentivar a participação dos estudantes em uma perspectiva construtivista e autônoma (COGO et al., 2009). O docente pode tirar partido dos mapas conceituais como instrumentos de organização/ delineamento curricular, para introduzir conteúdos ou para avaliar os conhecimentos (GREENBERG; WILNER, 2015). Ao longo do curso, podem ser planejados para uma disciplina, um tópico específico ou para o desenvolvimento de todo o programa educacional (MOREIRA, 1992). Estudantes do ensino superior têm sido beneficiados pelo uso dessa importante ferramenta que promove o pensamento crítico, potencializando a capacidade para a resolução de problemas e tomada de decisões, habilidades necessárias para a prática profissional. O pensamento crítico é um processo ativo na medida em que são avaliadas todas as evidências apresentadas antes de se decidir pela conduta a ser tomada (GUL; BOMAN, 2006). Os mapas conceituais podem ser concebidos em softwares que facilitam a sua construção como CMap Tools, com o qual é possível associar imagens, vídeos e URLs sendo facilmente compartilhados de forma síncrona

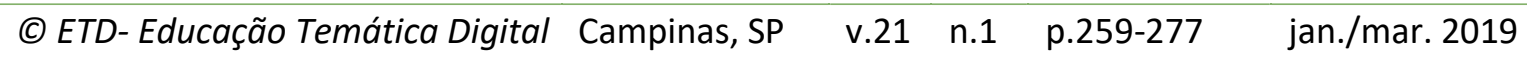


ou assíncrona (NOVAK, 2003). No formato digital, tornam-se um recurso adequado para o ensino presencial, para a educação a distância e em ambientes virtuais de aprendizagem (COGO et al., 2009). Assim, levando-se em conta o potencial dos mapas conceituais como facilitadores do processo de aprendizagem, pretende-se reportar seus efeitos nas diferentes áreas de conhecimento no ensino superior. A partir das publicações no período de 1992 a 2017, indicamos as aplicações didático-pedagógicas, contribuições no desenvolvimento de habilidades e desafios desta importante ferramenta para a promoção da aprendizagem significativa.

\section{MÉTODOS}

A presente pesquisa consiste na realização de uma revisão sistemática da literatura (RSL) (GOUGH; OLIVER; THOMAS, 2012) na qual foram analisadas as estratégias pedagógicas com os mapas conceituais em programas curriculares do ensino superior. As seguintes questões norteadoras foram formuladas: Quais os efeitos da utilização dos mapas conceituais para a aprendizagem no ensino superior? Como os mapas conceituais são utilizados e para quais finalidades no ensino superior? Para efeitos da RSL foram incluídos os artigos disponibilizados nas bases de dados entre 1992 e 2017: Academic OneFile; Academic Search Complete; Business Source Complete; CINAHL; CINAHL Plus; Directory of Open Access Journals; ERIC; MEDLINE; Psychology and Behavioral Sciences Collection; RCAAP; Science Citation Index; ScienceDirect; Scopus; Social Sciences Citation Index. Foram considerados artigos em português, inglês e espanhol. Para a busca foram definidas as seguintes palavras-chave: "mapas conceituais"/ "concept mapping"/ "mapas conceptuales" e "aprendizagem significativa" / "meaningful learning" / "aprendizage significativo" e "ensino superior" /" higher education"/ "enseñanza superior" ou "faculdade"/ "college"/ "universidad" ou "universidade"/ "university". Foram selecionados os artigos científicos escritos nos idiomas mencionados e disponíveis na íntegra online. Em um primeiro levantamento, foram encontrados 2620 artigos. Após realizada uma análise inicial de adequação, 419 artigos indicaram possibilidades em contribuir para a pesquisa. Entretanto, ao se tratar de uma SRL o pesquisador deve certificar-se de que sejam incluídas todas as publicações relevantes, além de garantir a exclusão daquelas que não atendam aos objetivos da pesquisa (SAMPAIO; MANCINI, 2007). Em seguida, realizou-se a análise dos trabalhos selecionados, tendo por critérios de inclusão a) a aplicação dos mapas conceituais no ensino superior e b) as finalidades e a forma de operacionalização dos mapas conceituais no ensino superior. Em seguida, foi realizada uma leitura integral de todos os artigos selecionados de forma a garantir que cumpriam os critérios de inclusão. Os artigos que cumpriram os critérios foram selecionados, sendo excluídos 351 e permanecendo 68 artigos. Os principais motivos das exclusões foram: o mesmo artigo publicado em duas bases de dados distintas, artigos que avaliavam os mapas conceituais em outros níveis de ensino, que não o ensino superior, e artigos que não descreviam adequadamente os procedimentos de aplicação dos mapas conceituais.

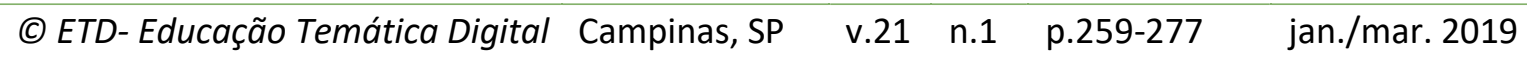




\section{RESULTADOS}

O presente estudo apresenta um número crescente de publicações que envolveram a utilização dos mapas conceituais, cobrindo várias áreas de conhecimento no ensino superior de 1992 a 2017, num total de 68 publicações (Quadro 1).

QUADRO 1. A produção de trabalhos publicados sobre mapas conceituais em diferentes áreas do conhecimento no ensino superior de 1992 a 2017.

\begin{tabular}{|l|l|c|}
\hline Período & Áreas do conhecimento & Total \\
\hline 1992-1999 & Enfermagem; Física. & 4 \\
\hline 2000-2009 & $\begin{array}{l}\text { Ciências Biológicas; Ciências da Saúde; Educação; Enfermagem; Farmácia; Física; } \\
\text { Medicina; Química. }\end{array}$ & 28 \\
\hline 2010-2017 & $\begin{array}{l}\text { Administração; Biblioteconomia, Bioquímica; Ciência da Computação; Ciências } \\
\text { Contábeis; Ciências Sociais; Direito; Educação; Enfermagem; Engenharia Química; } \\
\text { Engenharias; Farmácia; Letras; Medicina; Nutrição. }\end{array}$ & 36 \\
\hline
\end{tabular}

Fonte: as autoras (2018)

Particularmente no período de 2010 a 2017, observa-se não somente um maior número de publicações mas também uma maior diversidade das áreas do conhecimento que utilizaram os mapas conceituais para a promoção da aprendizagem.

\subsection{Finalidades da utilização dos mapas conceituais}

Os mapas conceituais podem ser trabalhados, no âmbito do ensino superior, para diferentes finalidades (Quadro 2).

QUADRO 2. Finalidades dos mapas conceituais no ensino superior e respectivas áreas do conhecimento.

\begin{tabular}{|l|l|c|}
\hline \multicolumn{1}{|c|}{ Finalidades } & \multicolumn{1}{|c|}{ Áreas do conhecimento } & Frequência \\
\hline $\begin{array}{l}\text { a) Promover a aprendizagem dos } \\
\text { conteúdos }\end{array}$ & $\begin{array}{l}\text { Administração; Biblioteconomia; Ciência da Computação; } \\
\text { Ciências Biológicas; Ciências Contábeis; Ciências da Saúde; } \\
\end{array}$ & $\begin{array}{l}\text { Educação; Enfermagem; Engenharia Química; Farmácia; } \\
\text { Física; Medicina; Nutrição; Química. }\end{array}$ \\
\hline b) Desenvolver o pensamento crítico & Enfermagem. & 11 \\
\hline c) Relacionar teoria e prática & Enfermagem; Medicina. & 9 \\
\hline d) Ajudar a resolver problemas & Enfermagem; Medicina. & 3 \\
\hline
\end{tabular}

Fonte: As autoras (2018)

a) Promover a aprendizagem significativa dos conteúdos:

Ao construírem os mapas conceituais, os estudantes são estimulados a integrarem seus conhecimentos de forma a ultrapassar a simples memorização e alcançar a aprendizagem significativa (HILL, 2004). Os mapas conceituais são utilizados em diferentes contextos na sala de aula como instrumentos facilitadores da aprendizagem significativa. Eles

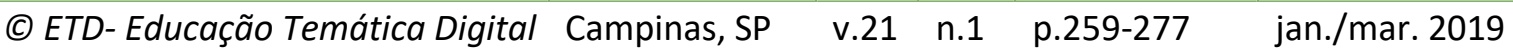


podem ser usados para verificar e acompanhar a aprendizagem sendo importantes sinalizadores dos conceitos aprendidos pelos estudantes sobre um determinado tema, disciplina ou texto científico. A aprendizagem dos conteúdos pode ser evidenciada nos mapas pela organização dos conceitos e qualidade das suas relações. Os estudantes podem elaborar um ou mais mapas no final das atividades ou ao longo do curso ou disciplina. Os estudantes manifestaram-se positivamente sobre a aplicabilidade dos mapas conceituais como ferramentas de aprendizagem. Estudos demonstraram que com a prática, os estudantes construíram mapas cada vez mais elaborados (ALMEIDA; MOREIRA, 2008; AZEVEDO et al., 2004; BELLUZZO; KOBAYASHI; FERES, 2004; BES PIÁ; BLASCO-TAMARITI; MUÑOZ-PORTERO, 2011; BHUSNURMATH et al., 2017; BITTENCOURT et al., 2013; CARABETTA JÚNIOR, 2013; CARR-LOPEZ et al., 2014; COGO et al., 2009; CUTRER et al., 2011; DUARTE; LOUREIRO; ZUKOWSKY-TAVARES, 2017; ERDEM; YILMAZ; OSKAY, 2009; FARIAS; FARIAS, 2016; GONZÁLEZ GARCÍA et al., 2012; GONZÁLEZ et al., 2008; HSU; HSIEH, 2005; KATILIUTE; DAUNORIENĖ, 2011; RAHNAMA; MARDANI-HAMOOLEH, 2017; RICART; ESTRADA, 2016; RUIZ-MORENO et al., 2007; WILGIS; MCCONNELL, 2008).

b) Desenvolver o pensamento crítico:

Os mapas conceituais demonstraram ser uma ferramenta efetiva para auxiliar os estudantes a desenvolverem o pensamento crítico que envolve a capacidade de refletir, inquirir e tomar decisões assertivas (ALL; HAVENS, 1997; LEE et al., 2013). Em estudos realizados com estudantes da área da saúde, os autores descrevem as atividades nas quais os mapas conceituais promovem o pensamento crítico tornando os estudantes mais aptos para as práticas clínicas (ALL; HUYCKE, 2007; ALL; HUYCKE; FISHER, 2003; CHEN et al., 2011; GUL; BOMAN, 2006; RAHNAMA; MARDANI-HAMOOLEH, 2017; SENITA, 2008; VACEK, 2009; WHEELER; COLLINS, 2003; WILGS; MCCONNELL, 2008).

c) Relacionar teoria e prática:

Mapas conceituais auxiliam os estudantes a associarem um novo conhecimento ao conhecimento preexistente. Durante a elaboração dos mapas, os estudantes da área de saúde realizam a integração dos conceitos básicos com as questões clínicas (GERDEMAN; LUX; JACKO, 2013; HARRISON; GIBBONS, 2013; VERONESE et al., 2013). O uso de mapas conceituais no contexto clínico tem auxiliado os estudantes a conectar o conhecimento teórico com a prática clínica (ALL; HAVENS, 1997; DALEY; TORRE, 2010; HICKS-MOORE, 2005; TORRE et al., 2007; WHEELER; COLLINS, 2003). O processo da construção dos mapas conceituais permite ao estudante refletir sobre a sua aprendizagem e a desenvolver o julgamento clínico (KADDOURA et al., 2016).

d) Ajudar a resolver problemas:

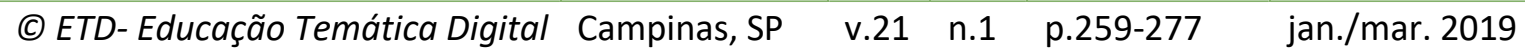


Ao resolver um problema, é necessário que o estudante lembre, transfira e aplique o conhecimento. Os mapas conceituais e a aprendizagem baseada em problemas são técnicas utilizadas para promover a aprendizagem ativa e significativa. Estudantes que trabalharam com mapas conceituais obtiveram um melhor desempenho na resolução de problemas quando comparados com estudantes que tiveram aulas tradicionais (GONZÁLEZ et al., 2008). Addae, Wilson e Carrington (2012) verificaram as percepções dos estudantes de Medicina sobre a aplicação de um método convencional de aprendizagem baseada em problemas em comparação com o método modificado de aprendizagem baseada em problemas com os mapas conceituais. Foi verificado que o método de aprendizagem baseada em problemas com mapas conceituais apresentou resultados significativamente melhores nos quatro domínios avaliados: afetivo, cognitivo, metacognitivo e interpessoal quando comparado ao método tradicional de aprendizagem baseada em problemas. Mapas conceituais demonstraram-se efetivos para representar o conhecimento, estimular a participação e a criatividade de estudantes de Enfermagem (CHAN, 2017).

É importante que durante o processo de formação o estudante desenvolva competências que o torne capaz de refletir, ser um sujeito ativo e crítico sobre o seu percurso de aprendizagem (FARIAS; FARIAS, 2016).

\subsection{Operacionalização dos mapas conceituais}

No quadro 3 estão indicados modos de operacionalização dos mapas conceituais no ensino superior. 
QUADRO 3. Modos de operacionalização dos mapas conceituais

\begin{tabular}{|c|c|c|c|}
\hline \multicolumn{2}{|c|}{ Modos de operacionalização } & Áreas do conhecimento & Frequência \\
\hline \multirow[t]{3}{*}{$\begin{array}{l}\text { a) Autoria dos mapas } \\
\text { conceituais }\end{array}$} & Estudante & $\begin{array}{l}\text { Biblioteconomia; Ciência da Computação; } \\
\text { Ciências Contábeis; Direito; Educação; } \\
\text { Enfermagem; Engenharias; Farmácia; } \\
\text { Física; Letras; Medicina; Nutrição; } \\
\text { Química;. }\end{array}$ & 29 \\
\hline & Professor & Ciências Contábeis. & 1 \\
\hline & $\begin{array}{l}\text { Estudante e } \\
\text { professor }\end{array}$ & $\begin{array}{l}\text { Administração; Bioquímica; Ciência da } \\
\text { Computação; Engenharia Química; } \\
\text { Medicina }\end{array}$ & 6 \\
\hline \multirow[t]{3}{*}{$\begin{array}{l}\text { b) Ambientes para } \\
\text { criar mapa } \\
\text { conceituais }\end{array}$} & Sala de aula & $\begin{array}{l}\text { Administração; } \text { Biblioteconomia; } \\
\text { Bioquímica; Ciência da Computação; } \\
\text { Ciências Biológicas; Ciências Contábeis; } \\
\text { Educação; Farmácia; Física; Medicina; } \\
\text { Engenharia Química; Engenharias; } \\
\text { Enfermagem; Química. }\end{array}$ & 25 \\
\hline & Extraclasse & Farmácia & 2 \\
\hline & Online & $\begin{array}{l}\text { Direito; } \quad \text { Educação; } \quad \text { Enfermagem; } \\
\text { Engenharias; Letras; Medicina. }\end{array}$ & 8 \\
\hline \multirow{3}{*}{$\begin{array}{l}\text { c) Utilizar diferentes } \\
\text { recursos para a } \\
\text { aplicação dos mapas } \\
\text { conceituais }\end{array}$} & Papel e lápis & Educação; Enfermagem. & 2 \\
\hline & $\begin{array}{l}\text { Recursos } \\
\text { digitais }\end{array}$ & $\begin{array}{l}\text { Administração; Bioquímica; Ciência da } \\
\text { Computação; Ciências Biológicas; Ciências } \\
\text { Contábeis; } \quad \text { Direito; Educação; } \\
\text { Enfermagem; Engenharia Química; } \\
\text { Engenharias; Farmácia; Física; Letras; } \\
\text { Medicina; Nutrição; Química. }\end{array}$ & 26 \\
\hline & $\begin{array}{l}\text { Papel e lápis e } \\
\text { recursos digitais }\end{array}$ & Ciências Contábeis; Farmácia; Medicina. & 3 \\
\hline \multirow{3}{*}{$\begin{array}{l}\text { d) Possibilitar a } \\
\text { experiência do } \\
\text { trabalho individual } \\
\text { ou em equipe }\end{array}$} & Individual & $\begin{array}{l}\text { Ciências Biológicas; Ciências da Saúde } \\
\text { Educação; Enfermagem; Medicina; } \\
\text { Nutrição. }\end{array}$ & 5 \\
\hline & Equipe & $\begin{array}{l}\text { Bioquímica; Ciência da Computação; } \\
\text { Ciências Contábeis; Ciências Sociais; } \\
\text { Direito; Educação; Enfermagem; } \\
\text { Engenharias; Farmácia; Medicina. }\end{array}$ & 19 \\
\hline & $\begin{array}{l}\text { Individual e } \\
\text { equipe }\end{array}$ & $\begin{array}{l}\text { Administração; Ciência da Computação; } \\
\text { Enfermagem; Engenharia Química; Física. }\end{array}$ & 5 \\
\hline \multicolumn{2}{|c|}{ e) Melhor performance nos exames } & $\begin{array}{l}\text { Administração; Bioquímica; Ciência da } \\
\text { Computação; Ciências Contábeis; } \\
\text { Enfermagem; Engenharia Química; Física; } \\
\text { Medicina; Nutrição. }\end{array}$ & 10 \\
\hline \multicolumn{2}{|c|}{$\begin{array}{l}\text { f) Detectar dificuldades na } \\
\text { aprendizagem }\end{array}$} & $\begin{array}{l}\text { Ciência da Computação; Enfermagem; } \\
\text { Física; Medicina. }\end{array}$ & 6 \\
\hline
\end{tabular}

Fonte: As autoras (2018) 
São várias as possibilidades de se operacionalizar atividades com os mapas conceituais, a seguir destacamos:

a) Mapas conceituais podem ser criados por professores e/ou estudantes:

Os mapas conceituais podem ser criados por estudantes como ferramentas que facilitam a representação dos conteúdos aprendidos (ADDAE; WILSON; CARRINGTON, 2012; ALMEIDA; MOREIRA, 2008; AZEVEDO et al., 2004; BHUSNURMATH et al., 2017; BITTENCOURT et al., 2013; CARABETTA JÚNIOR, 2013; CARR-LOPEZ et al., 2014; CHAN, 2017; COGO et al., 2009; DINARVAND; VAISI-RAYGANI, 2013; DUARTE; LOUREIRO; ZUKOWSKY-TAVARES, 2017; ERDEM; YILMAZ; OSKAY, 2009; FARIAS; FARIAS, 2016; GONZÁLEZ et al., 2008; HARRISON; GIBBONS, 2013; HILL, 2004; HSU; HSIEH, 2005; KALIUTE; DAUNORIENE், 2011; KREPSKY; MORASTONI; GOETTEN, 2014; LEAUBY; SZABAT; MAAS, 2010; RICART; ESTRADA, 2016; RUIZMORENO et al., 2007; SENITA, 2008; TORRE et al., 2007; TSENG et al., 2013; VIOLANTE; VEZZETTI, 2014; WANG et al., 2017; WU; CHEN; HOU, 2016; YEN; LEE; CHEN, 2012) e podem também ser criados pelos professores para apresentar e organizar os conteúdos de forma lógica, com o objetivo de melhorar a aprendizagem dos estudantes de um curso de Contabilidade (GREENBERG; WILNER, 2015). Mapas conceituais podem ainda ser construídos, inicialmente, pelo professor e posteriormente finalizados pelos estudantes. Mapas conceituais pré-construídos foram bem aceitos pelos estudantes, especialmente pelos inexperientes (BES PIÁ; BLASCO-TAMARITI; MUÑOZ-PORTERO, 2011; CUTRER et al., 2011; GONZÁLEZ GARCÍA et al., 2012; HO; KUMAR; VELAN, 2014; MARÉE; VAN BRUGGEN; JOCHEMS, 2013; SAEIDIFARD et al., 2014).

b) Aplicar mapas conceituais em diferentes ambientes:

Mapas conceituais podem ser aplicados na sala de aula (ADDAE; WILSON; CARRINGTON, 2012; ALMEIDA; MOREIRA, 2008; AZEVEDO et al., 2004; BES PIÁ; BLASCO-TAMARITI; MUÑOZPORTERO, 2011; BITTENCOURT et al., 2013; CARABETTA JÚNIOR, 2013; CHAN, 2017; ERDEM; YILMAZ; OSKAY, 2009; FARIAS; FARIAS, 2016; GONZÁLEZ GARCÍA et al., 2012; GONZÁLEZ et al., 2008; GREENBERG; WILNER, 2015; HARRISON; GIBBONS, 2013; HILL, 2004; HSU; HSIEH, 2005; KALIUTE; DAUNORIENĖ, 2011; LEAUBY; SZABAT; MAAS, 2010; MARÉE; VAN BRUGGEN; JOCHEMS, 2013; RICART; ESTRADA, 2016; RUIZ-MORENO et al., 2007; SAEIDIFARD et al., 2014; SENITA, 2008; TORRE et al., 2007; VIOLANTE; VEZZETTI, 2014; YEN; LEE; CHEN, 2012) ou nas atividades extraclasse (CARR-LOPEZ et al., 2014; DINARVAND; VAISI-RAYGANI, 2013) resultando em uma aprendizagem significativa, melhor desempenho acadêmico e ainda como instrumento para auxiliar o professor na identificação das dificuldades de aprendizagem dos conteúdos. Os mapas conceituais podem também ser incorporados para integrar o ensino, aprendizagem e colaboração em ambientes online (COGO et al., 2009; DALEY; CAÑAS; STARK-SCHWEITZER, 2007; HAY et al. 2008; HO; KUMAR; VELAN, 2014; 
KREPSKY; MORASTONI; GOETTEN, 2014; TSENG et al., 2013; WANG et al., 2017; WU; CHEN; HOU, 2016).

c) Utilizar diferentes recursos para a aplicação dos mapas conceituais:

Mapas conceituais podem ser criados manualmente, usando papel e lápis (RICART; ESTRADA, 2016; SENITA, 2008), ou utilizando recursos digitais (ADDAE; WILSON; CARRINGTON, 2012; ALMEIDA; MOREIRA, 2008; AZEVEDO et al., 2004; BES PIÁ; BLASCO-TAMARITI; MUÑOZPORTERO, 2011; BITTENCOURT et al., 2013; CARABETTA JÚNIOR, 2013; CARR-LOPEZ et al., 2014; COGO et al., 2009; GONZÁLEZ et al., 2008; GREENBERG; WILNER, 2015; HARRISON; GIBBONS, 2013; HILL, 2004; HO; KUMAR; VELAN, 2014; HSU; HSIEH, 2005; KREPSKY; MORASTONI; GOETTEN, 2014; MARÉE; VAN BRUGGEN; JOCHEMS, 2013; RUIZ-MORENO et al., 2007; TORRE et al., 2007; TSENG et al., 2013; WANG et al., 2017; WU; CHEN; HOU, 2016; YEN; LEE; CHEN, 2012). Os estudantes indicaram maior preferência na realização dos mapas com recursos digitais (LEAUBY; SZABAT; MAAS, 2010). É possível que o uso da tecnologia seja um fator que promova o envolvimento e estimule a criatividade dos estudantes principalmente quando constroem mapas com imagens em vez de somente textos (ERDEM; YILMAZ; OSKAY, 2009). Mapas conceituais podem ainda ser criados com imagens em três dimensões e neste caso, auxiliam os estudantes que possuem pouca habilidade espacial (VIOLANTE; VEZZETTI, 2014).

d) Possibilitar a experiência do trabalho individual ou em equipe:

Os mapas conceituais podem ser trabalhados em equipe (ADDAE; WILSON; CARRINGTON 2012; AYDOGAN; ERGUN, 2016; CARABETTA JÚNIOR, 2013; GONZÁLEZ et al., 2008; HARRISON; GIBBONS, 2013; HO; KUMAR; VELAN, 2014; HSU; HSIEH, 2005; KREPSKY; MORASTONI; GOETTEN, 2014; MARÉE; VAN BRUGGEN; JOCHEMS, 2013; TSENG et al., 2013; WU; CHEN; HOU, 2016; YEN; LEE; CHEN, 2012) ou individualmente (AZEVEDO et al., 2004; KADDOURA et al., 2016; RICART; ESTRADA, 2016; RUIZ-MORENO et al., 2007; TORRE et al., 2007), sendo que na maioria dos casos, os estudantes que construíram os mapas de forma colaborativa obtiveram melhores resultados na aprendizagem (KINCHIN; HAY, 2005). Segundo Cogo e colaboradores (2009), a reflexão gerada entre os estudantes durante a elaboração dos mapas sinaliza o potencial de produção coletiva que essa técnica possui seja no ensino presencial ou a distância. Mapas conceituais trabalhados colaborativamente podem auxiliar os estudantes a refletir e partilhar suas opiniões, resultando na construção do conhecimento. Em grupos, os estudantes são encorajados a se comunicarem, partilharem suas dúvidas e corrigirem os equívocos enquanto estão a construir os mapas (HSU; HSIEH, 2005). A construção colaborativa de mapas conceituais demonstrou ser uma experiência positiva ao estimular a participação e envolvimento dos estudantes, resultando em uma melhor performance nos exames (GONZÁLEZ GARCÍA et al., 2012). Mapas conceituais construídos de forma colaborativa em ambiente online foram efetivos para a promoção da aprendizagem e interação entre os estudantes nas dimensões cognitivas, metacognitivas e

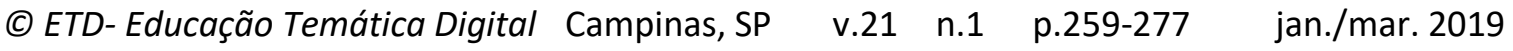


motivacionais-emocionais (WANG et al., 2017). Na sala de aula, a construção de mapas conceituais auxilia os estudantes a entender as vantagens do trabalho colaborativo e a ter a experiência de trabalho em equipe (HILL, 2004; KALIUTE; DAUNORIENE், 2011). As discussões promovem nos estudantes a autoavaliação e contribuem para o desenvolvimento do julgamento clínico (GERDEMAN; LUX; JACKO, 2013).

e) Melhor performance nos exames:

De acordo com David Jonassen (2007, p. 77): “Os alunos que estudam desta forma [mapas conceituais] irão, definitivamente, obter uma pontuação melhor em praticamente qualquer tipo de teste que o professor lhes dê". Ao facilitar a aprendizagem significativa, os mapas conceituais podem ser usados como uma estratégia que permite aos estudantes obter melhor desempenho nos exames ou testes. Nos cursos de Bioquímica, Engenharia, Física e Medicina, estudantes que trabalharam com mapas conceituais obtiveram melhor performance no exame final (ALMEIDA; MOREIRA, 2008; BES PIÁ; BLASCO-TAMARITI; MUÑOZ-PORTERO, 2011; BHUSNURMATH et al., 2017; GONZÁLEZ GARCÍA et al., 2012; MARÉE; VAN BRUGGEN; JOCHEMS, 2013; VERONESE et al., 2013). Todavia, em estudos realizados com estudantes de Medicina, não foram detectadas diferenças estatisticamente significativas nos resultados dos exames de múltipla escolha entre grupos que utilizaram mapas conceituais e grupos que não os utilizaram. Apesar dos exames de múltipla escolha serem muito utilizados para avaliar os estudantes, outros tipos de testes devem ser considerados para se detectar os efeitos dos mapas conceituais na aprendizagem e obter resultados diferentes (GONZÁLEZ et al., 2008; LEAUBY; SZABAT; MAAS, 2010). A aprendizagem significativa proporciona ao estudante uma maior retenção do conhecimento aumentando a sua capacidade para o aprendizado subsequente e futuro (NOVAK; GOWIN, 1984). Após as atividades com mapas conceituais, os estudantes demonstraram uma maior retenção dos conceitos e suas relações (CUTRER et al., 2011). Mapas conceituais foram utilizados em um curso de Tecnologia da Informação para auxiliar os estudantes na compreensão e revisão dos conteúdos. Nesse estudo, os estudantes utilizaram os mapas conceituais construídos durante o curso para se prepararem para o exame final, resultando em um melhor rendimento acadêmico (AYDOGAN; ERGUN, 2016).

f) Identificar as dificuldades na aprendizagem:

Ao serem usados para representarem o conhecimento adquirido, os mapas conceituais são para o professor uma importante ferramenta para auxiliá-lo na identificação de equívocos e dificuldades na aprendizagem, possibilitando esclarecer as dúvidas e fazer correções (ALMEIDA; MOREIRA, 2008; DALEY; TORRE, 2010; GONZÁLEZ GARCÍA et al., 2012; HSU; HSIEH, 2005; TORRE et al., 2007). O feedback proporcionado pelo uso dos mapas conceituais em ambiente online ajudou o docente a manter o entusiasmo e o interesse dos estudantes nos tópicos estudados (HO; KUMAR; VELAN, 2014).

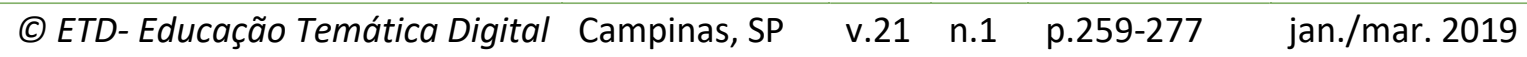


3.3. Dificuldades-na realização dos mapas conceituais

Embora a contribuição dos mapas conceituais seja notória para a aprendizagem, algumas limitações dessa ferramenta merecem ser consideradas (Quadro 4).

QUADRO 4. Dificuldades encontradas pelos estudantes do ensino superior durante a construção dos mapas conceituais.

\begin{tabular}{|l|l|c|}
\hline Dificuldades & Áreas do conhecimento & Frequência \\
\hline a) Estabelecimento de conceitos e ligações & $\begin{array}{l}\text { Biblioteconomia; Educação; Enfermagem; } \\
\text { Engenharia Química; Medicina. }\end{array}$ & 8 \\
\hline $\begin{array}{l}\text { b)Tempo para a elaboração dos mapas } \\
\text { conceituais }\end{array}$ & $\begin{array}{l}\text { Enfermagem; Engenharia Química; } \\
\text { Medicina. }\end{array}$ & 5 \\
\hline c) Resistência dos estudantes & $\begin{array}{l}\text { Ciência da Computação; Enfermagem; } \\
\text { Medicina. }\end{array}$ & 2 \\
\hline d) Integração da tecnologia & Direito; Enfermagem. & 1 \\
\hline e) Inserção de atividades extracurriculares & Física. & 4 \\
\hline
\end{tabular}

Fonte: As autoras (2018)

É necessário conhecer as dificuldades encontradas pelos estudantes ao desenvolverem atividades com mapas conceituais bem como buscar meios de amenizá-las ou superá-las.

a) Estabelecimento de conceitos e ligações:

Problemas na categorização de conceitos e seleção adequada das suas ligações foram algumas dificuldades observadas pelos estudantes durante a elaboração dos mapas conceituais (FARIAS; FARIAS, 2016; GONZÁLEZ et al., 2008). Wu, Chen e Hou (2016) mostraram que pode ser mais fácil para os estudantes definir um nó (conceito) do que definir suas conexões. No entanto, quando há muitos conceitos torna-se mais difícil conectá-los (BES PIÁ; BLASCO-TAMARITI; MUÑOZ-PORTERO, 2011). As principais preocupações dos estudantes com mapas conceituais estavam relacionadas à dificuldade na organização espacial, o que resultou em alguns produtos finais desordenados que eram difíceis de interpretar e rever (VERONESE et al., 2013). A frustração dos estudantes em relação ao mapas conceituais poderia ser reduzida com a presença de um instrutor disponível para consultas e em ambientes que permitissem discussões entre os pares (GERDEMAN; LUX; JACKO, 2013; HARRISON; GIBBONS, 2013; TORRE et al., 2007).

b) Tempo para a elaboração dos mapas conceituais:

De acordo com relatos dos estudantes, um aspecto desfavorável dos mapas conceituais refere-se ao tempo necessário para a sua elaboração. Os estudantes sentiram que as atividades para a elaboração dos mapas conceituais eram muito demoradas e aumentaram

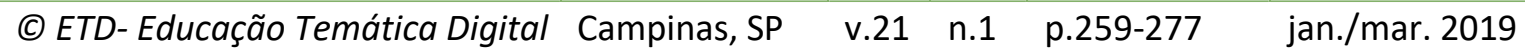


sua carga de trabalho acadêmico (BES PIÁ; BLASCO-TAMARITI; MUÑOZ-PORTERO, 2011; HARRISON; GIBBONS, 2013; SENITA, 2008; VERONESE et al., 2013). No entanto, a maioria dos estudantes percebeu que o tempo necessário para a criação dos mapas conceituais diminui à medida que adquiriam experiência (HARRISON; GIBBONS, 2013). Ao usarem mapas conceituais online pré-construídos com feedback associado, os estudantes demonstraram uma melhor aceitação por esse método que permitiu reduzir o tempo para a elaboração dos mapas (HO; KUMAR; VELAN, 2014).

c) Resistência dos estudantes:

Em alguns estudos, foi observada uma resistência dos estudantes ao uso de mapas conceituais. A resistência na elaboração dos mapas conceituais ocorreu provavelmente devido à dificuldade de alguns estudantes em assumir um papel mais ativo em sua própria aprendizagem (GONZÁLEZ et al., 2008). Ademais, os estudantes expressaram sentimentos negativos sobre os mapas conceituais por não entenderem completamente os seus benefícios (HARRISON; GIBBONS, 2013). No entanto, à medida que os estudantes se tornam mais confiantes com o método, a resistência tende a desaparecer (VERONESE et al., 2013). A resistência dos estudantes ao método tende a diminuir ao proporcionar um ambiente colaborativo e de trabalho em equipe (GONZÁLEZ GARCÍA et al., 2012).

d) Integração da tecnologia:

Outro desafio foi o uso do software para a construção dos mapas conceituais. Os estudantes expressaram não possuir o software em casa e não saber usá-lo. No entanto, a maioria dos estudantes manifestou que seus sentimentos negativos diminuíram à medida que suas habilidades aumentavam na elaboração dos mapas, juntamente com sua familiaridade com o software. Os estudantes sugeriram que seria importante oferecer um workshop inicial sobre os aspectos teóricos e técnicos dos mapas conceituais com duração suficiente para que os participantes pudessem praticar a criação de mapas conceituais eletrônicos (HARRISON; GIBBONS, 2013). Krepsky, Morastoni e Goetten (2014) relataram uma resistência inicial dos participantes quanto ao uso das tecnologias para a elaboração dos mapas conceituais sendo necessário um tempo maior para a adaptação e implementação da abordagem.

e) Inserção de atividades extracurriculares:

Desenvolver práticas que requeiram a participação de estudantes sem que as mesmas tenham sido previamente definidas no programa da disciplina foi uma dificuldade relatada em um estudo que envolveu a elaboração de mapas conceituais como atividade extracurricular. Há um risco de desistência ou falta de adesão dos estudantes às atividades para fins de pesquisa sem o seu planejamento prévio na disciplina ou curso (ALMEIDA; MOREIRA, 2008).

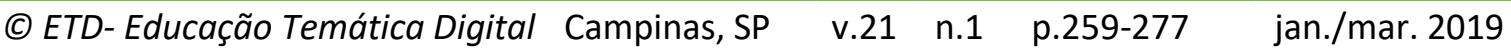


Assim, as atividades com mapas conceituais precisam ser bem planejadas, sendo avaliadas as características e necessidades dos estudantes a fim de se alcançar a aprendizagem pretendida.

\section{CONSIDERAÇÕES FINAIS}

Os mapas conceituais demonstraram ser uma estratégia de efetiva aprendizagem no ensino superior. Durante uma disciplina ou módulo, os estudantes universitários têm contato com vários conceitos e denominações que muitas vezes estão fragmentados e desconectados o que dificulta o entendimento do conteúdo. Ao praticar a organização e integração de vários conceitos durante a construção dos mapas, o estudante tem como resultado todo o conteúdo abordado em um formato esquemático que facilita a compreensão e revisão. Os mapas podem ser ampliados à medida que novos conteúdos são aprendidos. Assim, tornam-se estratégias de ensino muito positivas a serem aplicadas em um módulo ou ao longo de uma disciplina ou curso (GONZÁLEZ GARCÍA et al., 2012; RICART; ESTRADA, 2016). A partir da análise dos mapas conceituais é possível avaliar e acompanhar a aprendizagem. É possível relacionar uma progressiva qualidade dos mapas conceituais com uma melhor performance acadêmica dos estudantes ao longo do curso. Além de facilitar a correção de equívocos, esclarecimento de dúvidas e oferecer o contínuo feedback que é essencial para manter o entusiasmo e envolvimento dos estudantes. Mapas conceituais são também importantes ferramentas para auxiliar os estudantes na retenção dos conteúdos (BHUSNURMATH et al., 2017; HO; KUMAR; VELAN, 2014). Tornam-se estratégias ainda mais interessantes e estimulantes quando trabalhadas colaborativamente. As discussões, partilha de opiniões e negociações entre os colegas durante a elaboração dos mapas conceituais facilitam a compreensão do conteúdo levando os estudantes a uma experiência positiva na aprendizagem. A resistência dos estudantes ao método diminui quando estão envolvidos em atividades que favorecem a colaboração e o trabalho em grupo (COGO et al., 2009; GONZÁLEZ GARCÍA et al., 2012; WU; CHEN; HOU, 2016).

As atividades ainda são facilitadas com a utilização das tecnologias digitais que permitem a elaboração de mapas conceituais presencialmente ou à distância de forma colaborativa, além dos recursos tecnológicos permitirem a construção de mapas mais criativos e elaborados (COGO et al., 2009; WU; CHEN; HOU, 2016). Mapas conceituais contribuem para o desenvolvimento de habilidades importantes para uma adequada formação acadêmica e exercício profissional. O pensamento crítico é uma das habilidades promovidas com as práticas de mapas conceituais que auxiliam os estudantes a tornarem-se aptos para as atividades profissionais. Além disso, provaram ser uma ferramenta útil para associar teoria e prática, proporcionando a dinamização dos conteúdos e facilitando a contextualização dos mesmos (DALEY; TORRE, 2010; LEE et al., 2013; SENITA, 2008). 
Sobre as limitações referentes à elaboração dos mapas conceituais no ensino superior, os estudantes demonstraram dificuldades para a categorização dos conceitos e suas conexões, ocasionando um tempo maior que o necessário para a sua construção. Tais aspectos, se não ultrapassados, resultam em uma maior resistência e frustração dos estudantes em relação à abordagem. Vale ressaltar a importância da realização de uma capacitação inicial para os estudantes a fim de esclarecê-los sobre importância do método e de seus benefícios na aprendizagem, além de promover atividades prévias que permitam ao estudante praticar a construção dos mapas. No caso de se utilizar recursos tecnológicos, é conveniente verificar antes da sua implementação se todos os participantes são capazes de utilizá-los de forma adequada (FARIAS; FARIAS, 2016). Nota-se um maior envolvimento dos estudantes quando eles compreendem como executar as atividades com mapas conceituais e percebem seus benefícios na aprendizagem (HARRISON; GIBBONS, 2013).

Portanto, cada vez mais os mapas conceituais são usados no ensino superior com resultados positivos no processo de ensino-aprendizagem. Nos últimos anos, foram observados não só um maior número de trabalhos, mas também uma maior diversificação nas áreas do conhecimento que adotaram os mapas conceituais para múltiplas atividades em sala de aula. No entanto, estudos sobre a utilização dessa ferramenta para apoiar a aprendizagem dos estudantes universitários ao longo do curso ainda são escassos, urge, assim, avaliar seus efeitos a longo prazo, bem como as percepções dos estudantes. Nesse sentido, faz-se necessário incentivar cada vez mais docentes e estudantes para o uso dos mapas conceituais em suas atividades acadêmicas e assim promover uma aprendizagem significativa.

\section{REFERÊNCIAS}

ADDAE, Jonas Innies; WILSON, Jacqueline; CARRINGTON, Christine. Students' perception of a modified form of PBL using concept mapping. Medical Teacher, [s.I.], v. 34, n. 11, p. 756-762, nov. 2012.

AKINSANYA, Cynthia; WILLIAMS, Mriga. Concept mapping for meaningful learning. Nurse Education Today, [s.I.], v. 24, n. 1, p. 41-46, jan. 2004.

ALL, Anita; HAVENS, Robyn. Cognitive/concept mapping: a teaching strategy for nursing. Journal of Advanced Nursing, [s.I.], v. 25, n. 6, p. 1210-1219, jun. 1997.

ALL, Anita; HUYCKE, LaRae; FISHER, Mark. Instructional tools for nursing education: concept maps. Nursing Education Perspectives, [s.I.], v. 24, n. 6, p. 311-317, 2003.

ALL, Anita; HUYCKE, LaRae. Serial concept maps: tools for concept analysis. Journal of Nursing Education, [s.I.], v. 46, n. 5, p. 217-224, 2007. 
ALMEIDA, Voltaire; MOREIRA, Marco. Antônio. Mapas conceituais no auxílio à aprendizagem significativa de conceitos da óptica física. Revista Brasileira de Ensino de Física, São Paulo, v. 30, n. 4, p. 1-7, 2008.

AUSUBEL, David Paul. The use of advance organizers in the learning and retention of meaningful verbal material. Journal of Educational Psychology, [s.I.], v. 51, n. 5, p. 267272, 1960.

AYDOGAN, Tuncay; ERGUN, Serap. A study to determine the contribution made by concept maps to a computer architecture and organization course. European Journal of Contemporary Education, [s.I.], v. 15, n. 1, p. 76-83, mar. 2016.

AZEVEDO, Ana Maria Ponzio et al. Mapas conceituais e o jogo: estratégias pedagógicas de ensino e aprendizagem de Bioquímica. Informática na educação: teoria e prática. Porto Alegre, v. 7, n. 1, p. 59-71, 2004.

BELLUZZO, Regina Célia Baptista; KOBAYASHI, Maria Carmo Monteiro; FERES, Glória Georges. Information literacy: um indicador de competência para a formação permanente de professores na sociedade do conhecimento. Educação Temática Digital, Campinas, v. 6, n. 1, p. 81-99, dez. 2004.

BES PIÁ, Amparo; BLASCO-TAMARITI, Encarna; MUÑOZ-PORTERO, María José. Different applications of concept maps in higher education. Journal of Industrial Engineering and Management, [s.I.], v. 4, p. 81-102, 2011.

BHUSNURMATH, Shivayogi et al. Concept map as an adjunct tool to teach pathology. Indian Journal of Pathology and Microbiology, [s.I.], v. 60, n. 2, p. 226-231, abr. 2017.

BITTENCOURT, Greicy Kelly Gouveia Dias et al. Concept maps of the graduate programme in nursing: experience report. Revista Gaúcha de Enfermagem. Porto Alegre, v. 34, n. 2, p. 172-176, 2013.

CARABETTA JÚNIOR, Valter. A utilização de mapas conceituais como recurso didático para a construção e interrelação de conceitos. Revista Brasileira de Educação Médica. Rio de Janeiro, v. 37, n. 3, p. 441-447, 2013.

CARR-LOPEZ, Sian et al. The utility of concept maps to facilitate higher-level learning in a large classroom setting. American Journal of Pharmaceutical Education, [s.l.], v. 78, n. 9, p. 1-7, nov. 2014.

CHAN, Zenobia. A qualitative study on using concept maps in problem-based learning. Nurse Education in Practice, [s.I.], v. 24, p. 70-76, maio 2017.

CHEN, Shiah-Lian et al. Effects of concept map teaching on students' critical thinking and approach to learning and studying. Journal of Nursing Education, [s.I.], v. 50, n. 8, p. 466469, abr. 2011.

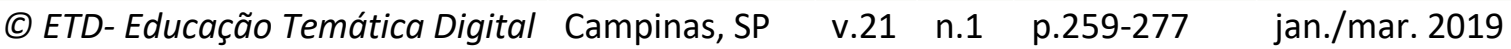


COGO, Ana Luísa Petersen et al. Avaliação de mapas conceituais elaborados por estudantes de enfermagem com o apoio de software. Texto \& Contexto Enfermagem, Florianópolis, v. 18, n. 3, p. 482-488, jul./set. 2009.

CUTRER, William et al. Use of an expert concept map as an advance organizer to improve understanding of respiratory failure. Medical Teacher, [s.I.], v. 33, n. 12, p. 1018-1026, dez. 2011.

DALEY, Barbara; CAÑAS, Alberto; STARK-SCHWEITZER, Tracy. CmapTools: integrating teaching, learning, and evaluation in online courses. New Directions for Adult and Continuing Education, [s.I.], v. 2007, n. 113, p. 37-47, 2007.

DALEY, Barbara; TORRE, Dario. Concept maps in medical education: an analytical literature review. Medical Education, [s.I.], v. 44, n. 5, p. 440-448, 2010.

DINARVAND, Gholamabbas; VAISI-RAYGANI, Assad. The effect of task-based teaching via drawing the concept map of metabolic pathways as homework on the academic achievement of pharmaceutical students in biochemistry course. Educational Research in Medical Sciences, [s.I.], v. 2, n. 1, p. 26-28, 2013.

DUARTE, Enios Carlos; LOUREIRO, Ana Cláudia; ZUKOWSKY-TAVARES, Cristina. Challenges and weaknesses in the use of concept maps as a learning strategy in undergraduate health programs. Knowledge Management \& E Learning, [s.I.], v. 9, n. 3, p. 380-391, 2017.

ERDEM, Emine; YILMAZ, Ayhan; OSKAY, Özge Özyalçın. The effect of concept mapping on meaningful learning of Atom and bonding. Procedia - Social and Behavioral Sciences, [s.I.], v. 1, n. 1, p. 1586-1590, 2009.

FARIAS, Maria Giovanna Guedes; FARIAS, Gabriela Belmont de. Aplicação de mapas conceituais como ferramentas didático-pedagógicas na área de recursos e serviços de informação. Biblios: Journal of Librarianship and Information Science, [s.I.], n. 63, p. 1327, jul. 2016.

GERDEMAN, Jaime; LUX, Kathleen; JACKO, Jean. Using concept mapping to build clinical judgment skills. Nurse Education in Practice, [s.I.], v. 13, n. 1, p. 11-17, jan. 2013.

GONZÁLEZ GARCÍA, Fermín María et al. Aplicación de mapas conceptuales en ingeniería en computación para evaluar aprendizaje significativo. Revista de Docencia Universitaria, [s.l.], v. 10, p. 459-475, 2012.

GONZÁLEZ, Hilda Leonor et al. Mediated learning experience and concept maps: a pedagogical tool for achieving meaningful learning in medical physiology students. Advances in Physiology Education, [s.I.], v. 32, n. 4, p. 312-316, dez. 2008.

GOUGH, David; OLIVER, Sandy; THOMAS, James. An introduction to systematic reviews. 2 ed. London: SAGE Publications, 2017. 352 p. 
GREENBERG, Rochelle Kaplan; WILNER, Neil. Using concept maps to provide an integrative framework for teaching the cost or managerial accounting course. Journal of Accounting Education, [s.I.], v. 33, n. 1, p. 16-35, mar. 2015.

GUL, Raisa; BOMAN, Jeanette. Concept mapping: a strategy for teaching and evaluation in nursing education. Nurse Education Practice, [s.I.], v. 6, p. 199-206, 2006.

HARRISON, Suzanne; GIBBONS, Caroline. Nursing student perceptions of concept maps: from theory to practice. Nursing Education Perspectives, [s.I.], v. 34, n. 6, p. 395-399, nov. 2013.

HAY, David et al. Measuring the quality of e-learning. British Journal of Educational Technology, [s.I.], v. 39, n. 6, p. 1037-1056, nov. 2008.

HICKS-MOORE, Sandee. Clinical concept maps in nursing education: an effective way to link theory and practice. Nurse Education in Practice, [s.I.], v. 5, n. 6, p. 348-352, nov. 2005.

HILL, Lilian. Concept mapping in a pharmacy communications course to encourage meaningful student learning. American Journal of Pharmaceutical Education, [s.l.], v. 68 , n. 5, p. 1-9, set. 2004.

HO, Veronica; KUMAR, Rakesh; VELAN, Gary. Online testable concept maps: benefits for learning about the pathogenesis of disease. Medical Education, [s.I.], v. 48, n. 7, p. 687697, jun. 2014

HSU, Liling; HSIEH, Suhing. Concept maps as an assessment tool in a nursing course. Journal of Professional Nursing, [s.I.], v. 21, n. 3, p. 141-149, mai. 2005.

JONASSEN, David. Computadores, ferramentas cognitivas: desenvolver o pensamento crítico nas escolas. Porto: Porto Editora, 2007. 320 p.

KADDOURA, Mahmoud et al. Impact of concept mapping on the development of clinical judgment skills in nursing students. Teaching and Learning in Nursing, [s.I.], v. 11, n. 3, p. 101-107, jul. 2016.

KATILIUTE, Egle.; DAUNORIENĖ, Asta. Toward meaningful learning in economics and management studies using concept maps in a quality management course. Economics \& Management, [s.I.], v. 16, p. 758-765, 2011.

KINCHIN, Ian; HAY, David. Using concept maps to optimize the composition of collaborative student groups: a pilot study. Journal of Advanced Nursing, [s.I.], v. 51, n. 2, p. 182-187, jul. 2005.

KREPSKY, Giselle Marie; MORASTONI, Eliana Pacheco; GOETTEN, Willian. Mapas conceituais na pesquisa do direito ambiental e na formação de professores. Interfaces Científicas Humanas e Sociais. Tiradentes, v. 3, n. 1, p. 55-65, 2014.

LEAUBY, Bruce; SZABAT, Kathryn; MAAS, Jayne. Concept mapping - an empirical study in introductory financial accounting. Accounting Education, [s.I.], v. 19, n. 3, p. 279-300, jun. 2010.

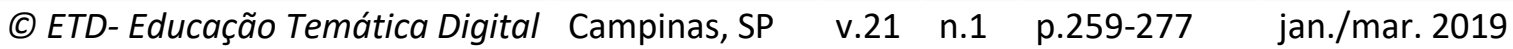


LEE, Weillie et al. The longitudinal effect of concept map teaching on critical thinking of nursing students. Nurse Education Today, [s.I.], v. 33, n. 10, p. 1219-1223, out. 2013.

MARÉE, Ton; VAN BRUGGEN, Jan; JOCHEMS, Wim. Effective self-regulated science learning through multimedia-enriched skeleton concept maps. Research in Science \& Technological Education, [s.l.], v. 31, n. 1, p. 16-30, abr. 2013.

MOREIRA, Marco Antônio. Mapas conceituais no ensino da Física. Textos de Apoio ao Professor de Física, Porto Alegre, n. 3, 1992.

NOVAK, Joseph. The promise of new ideas and new technology for improving teaching and learning. Cell Biology Education, [s.I.], v. 2, n. 2, p. 122-132, jun. 2003.

NOVAK, Joseph; GOWIN, Bob. Learning How to Learn. New York: Cambridge University, 1984. $216 \mathrm{p}$.

RAHNAMA, Farideh; MARDANI-HAMOOLEH, Marjan. Iranian nursing students' perceptions regarding use of concept mapping: a content analysis. Research \& Development in Medical Education, [s.l.], v. 6, n. 1, p. 45-50, 2017.

RICART, Maria; ESTRADA, Assumpta. Los conocimientos sobre la proporcionalidad de los futuros maestros a través del mapa conceptual. Indagatio Didactica, [s.I.], v. 8, n. 4, p. 3141, 2016.

RITCHHART, Ron; TURNER, Terri; HADAR, Linor. Uncovering students' thinking about thinking using concept maps. Metacognition and Learning, [s.I.], v. 4, n. 2, p. 145-159, jan. 2009.

RUIZ-MORENO, Lídia et al. Mapa conceitual: ensaiando critérios de análise. Ciência \& Educação, Bauru, v. 13, n. 3, p. 453-463, 2007.

SAEIDIFARD, Farzane et al. Concept mapping as a method to teach an evidence-based educated medical topic: a comparative study in medical students. Journal of Diabetes and Metabolic Disorders, [s.l.], v. 13, n. 1, p. 86-90, 2014.

SAMPAIO, Rosana Ferreira; MANCINI, Marisa Cotta. Estudos de revisão sistemática: um guia para síntese criteriosa da evidência científica. Revista Brasileira de Fisioterapia, São Carlos, v. 11, n. 1, p. 83-89, 2007.

SENITA, Julie. The use of concept maps to evaluate critical thinking in the clinical setting. Teaching and Learning in Nursing, [s.I.], v. 3, n. 1, p. 6-10, jan. 2008.

TORRE, Dario et al. A qualitative evaluation of medical student learning with concept maps. Medical Teacher, [s.I.], v. 29, n. 9-10, p. 949-955, jan. 2007.

TSENG, Kuo-Hung et al. Using creative problem solving to promote students' performance of concept mapping. International Journal of Technology and Design Education, [s.I.], v. 23, p. 1093-1109, 2013.

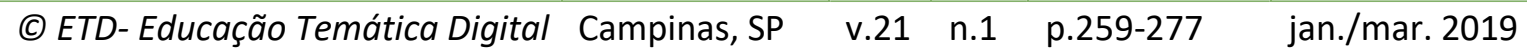


VACEK, Jenny. Using a conceptual approach with concept mapping to promote critical thinking. Journal of Nursing Education, [s.I.], v. 48, n. 1, p. 45-48, jan. 2009.

VERONESE, Carolina et al. A randomized pilot study of the use of concept maps to enhance problem-based learning among first-year medical students. Medical Teacher, [s.I.], v. 35, n. 9, p. 1478-1484, abr. 2013.

VIOLANTE, Maria Grazia; VEZZETTI, Enrico. Design of web-based interactive 3D concept maps: a preliminary study for an engineering drawing course. Computer Applications in Engineering Education, [s.I.], v. 23, n. 3, p. 403-411, set. 2014.

WANG, Minhong et al. The use of web-based collaborative concept mapping to support group learning and interaction in an online environment. The Internet and Higher Education, [s.I.], v. 34, p. 28-40, 2017.

WHEELER, Linda; COLLINS, Susan. The influence of concept mapping on critical thinking in baccalaureate nursing students. Journal of Professional Nursing, [s.I.], v. 19, n. 6, p. 339346, nov. 2003.

WILGIS, Michele; MCCONNELL, Joy. Concept mapping: an educational strategy to improve graduate nurses' critical thinking skills during a hospital orientation program. The Journal of Continuing Education in Nursing, [s.I.], v. 39, n. 3, p. 119-126, mar. 2008.

WU, Sheng-Vi; CHEN, Sherry Y.; HOU, Huei-Tse. Exploring the interactive patterns of concept map-based online discussion: a sequential analysis of users' operations, cognitive processing, and knowledge construction. Interactive Learning Environments, [s.I.], v. 24, n. 8, p. 1778-1794, jun. 2016.

YEN, Jung-Chuan; LEE, Chun-Yi; CHEN, I-Jung. The effects of image-based concept mapping on the learning outcomes and cognitive processes of mobile learners. British Journal of Educational Technology, [s.I.], v. 43, n. 2, p. 307-320, abr. 2012.

\section{AGRADECIMENTOS}

Ao CEIS20 e ao LabTE da Universidade de Coimbra pelo fomento à pesquisa.

Revisão gramatical do texto sob responsabilidade de: Estela Knitter Barros E-mail: estelaknitter@gmail.com 